\title{
Las tecnologías de la información y comunicación como herramienta de la metodología de la investigación
}

\section{Information and communication technologies as a tool for research methodology}

1 Marco Antonio Gavilanes Sagñay (iD) https://orcid.org/0000-0002-7470-3732 Escuela Superior Politécnica de Chimborazo (ESPOCH), Facultad de Administración de Empresas, Riobamba, Chimborazo, Ecuador,

marco.gavilanes@espoch.edu.ec

2 Willian Geovanny Yanza Chávez iD https://orcid.org/0000-0002-9688-7309 Escuela Superior Politécnica de Chimborazo (ESPOCH), Facultad de Administración de Empresas, Riobamba, Chimborazo, Ecuador willian.yanza@espoch.edu.ec

3 Johana Katherine Montoya Lunavictoria $\quad$ (iD) https://orcid.org/0000-0001-9138-0296 Universidad Nacional de Chimborazo (UNACH), Facultad de Ciencias de la Educación, Humanas y Tecnologías, Ecuador jhoana.montoya@unach.edu.ec

4 Luis Eulogio Benítez Cevallos https://orcid.org/0000-0002-7340-8205 Instituto Superior Tecnológico Carlos Cisneros, Riobamba, Chimborazo, Ecuador, luisbenitez2578@ hotmail.com

Artículo de Investigación Científica y Tecnológica Enviado: $10 / 12 / 2021$

Revisado: $25 / 12 / 2021$

Aceptado: 10/01/2021

Publicado:01/02/2022

DOI: https://doi.org/10.33262/ap.v4i1.135

Cítese:

Gavilanes Sagñay, M. A., Yanza Chávez, W. G., Montoya Lunavictoria, J. K., \& Benítez Cevallos, L. E. (2022). Las tecnologías de la información y comunicación como herramienta de la metodología de la investigación. AlfaPublicaciones, 4(1), 77-96. https://doi.org/10.33262/ap.v4i1.135

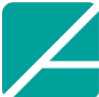

Ciencia Digital

ALFA PUBLICACIONES, es una Revista Multidisciplinar, Trimestral, que se publicará en soporte electrónico tiene como misión contribuir a la formación de profesionales competentes con visión humanística y crítica que sean capaces de exponer sus resultados investigativos y científicos en la misma medida que se promueva mediante su intervención cambios positivos en la sociedad. https://alfapublicaciones.com

La revista es editada por la Editorial Ciencia Digital (Editorial de prestigio registrada en la Cámara Ecuatoriana de Libro con No de Afiliación 663) www.celibro.org.ec 


\section{Palabras}

claves:

tecnología de la información y comunicación, metodología de la investigación, educación

superior.

\section{Keywords:}

information and communication technology, methodology of
Resumen

Introducción. La utilización de las Tecnologías de la Información y la Comunicación ha impactado positivamente en las diferentes esferas. Objetivo. Determinar la incidencia de la utilización de las Tecnologías de la Información y la Comunicación en la transformación de la Metodología de la Investigación Científica en el contexto de la Educación Superior. Metodología. Se utilizó una metodología descriptiva, basada en la Revisión Sistemática de la Literatura, apoyada en métodos teóricos y en la revisión documental mediante el establecimiento de criterios de búsqueda referenciales. Resultados. 1.Se evidencia que las TICs constituyen herramientas capaces de optimizar significativamente la calidad de la Metodología de la Investigación en el contexto de la Educación Superior, su empleo permite conseguir en menor tiempo y con la calidad requerida los objetivos planteados, creando espacios para el intercambio y la generación de soluciones. 2. Se demuestra que el desafío de la investigación en la universidad radica en el rol del docente que imparte la asignatura de Metodología de la Investigación, quien deberá tener las competencias que le permitan realizar una labor tutorial que congregue los saberes: teóricos, prácticos y pedagógicos y la interacción entre los investigadores. 3. Se sustenta que la información que brindan las técnicas cualitativas, utilizando las herramientas TICs, presentan tanta validez como las que aportan las técnicas cuantitativas. 4. Existe el riesgo por el investigador de enfocar su atención en el uso de las herramientas tecnológicas por encima del análisis metodológico de la investigación. Conclusiones. Se determina la alta incidencia de las Tecnologías de la Información y Comunicación en la transformación de la Metodología de la Investigación Científica en el contexto de la Educación Superior, su empleo constituye una revolución digital y metodológica en las investigaciones, sin embargo, su uso indiscriminado puede traer riesgos que deben ser controlados y evitados de forma permanente.

Introduction. The use of Information and Communication Technologies has had a positive impact in different spheres. Target. Determine the incidence of the use of Information and Communication Technologies in the transformation of the Scientific Research Methodology in the context of Higher 
the research, higher education
Education. Methodology. A descriptive methodology was used, based on the Systematic Review of the Literature, supported by theoretical methods and documentary review by establishing referential search criteria. Results. 1. It is evident that ICTs are tools capable of significantly optimizing the quality of Research Methodology in the context of Higher Education, their use allows the objectives set to be achieved in less time and with the required quality, creating spaces for exchange and the generation of solutions. 2. It is shown that the challenge of research at the university lies in the role of the teacher who teaches the subject of Research Methodology, who must have the skills that allow him to carry out tutorial work that brings together knowledge: theoretical, practical and pedagogical and interaction between researchers. 3 . It is argued that the information provided by qualitative techniques, using ICT tools, is as valid as that provided by quantitative techniques. 4. There is a risk for the researcher to focus his attention on the use of technological tools above the methodological analysis of the research. Conclusions. The high incidence of Information and Communication Technologies in the transformation of the Scientific Research Methodology in the context of Higher Education is determined, its use constitutes a digital and methodological revolution in research, however, its indiscriminate use can bring risks that must be controlled and avoided permanently.

\section{Introducción}

Las Tecnologías de la Información y la Comunicación (TICs) transformaron la manera en que nos relacionamos e interactuamos en la generalidad de los ámbitos de nuestra vida, y uno de los ejemplos más latente, es el aporte que realizan a la Investigación.

En este sentido, mediante las TICs se ha logrado el procesamiento y difusión de la información científica de una manera más efectiva, posibilitando el aumento del interés por parte de docentes, estudiantes y profesionales en la investigación, debido a una mayor accesibilidad por parte de la comunidad académica a la amplia red de conocimiento, con la utilización de múltiples canales de información, en especial de la internet. 
En referencia a lo anterior, Cisneros \& César (2003) manifiestan que la aplicación de las Nuevas Tecnologías de la Información (TIC), se encuentra materializado, entre otros elementos esenciales, en la difusión de programas específicos y en los resultados investigativos logrados mediante el apoyo de estas.

De igual forma, dado el desarrollo alcanzado por las TICs en la educación, el acceso a artículos científicos es mucho más abierto, tanto en las redes privadas de las universidades y centros científicos como en la propia web libre, donde se encuentran una gran variedad de documentos científicos de consulta libre que posibilitan el enriquecimiento de la labor científica y aumentando de manera significativa su efectividad.

Por otra parte, las TICs impulsa la retroalimentación de tópicos de investigación, así como permite contrastar resultados obtenidos de otros estudios realizados, favoreciendo la formulación de proyectos de investigación que incidirán en mayor medida en el campo de estudio respectivo, por lo que se evita la repetición de situaciones y problemas que ya han sido objeto de estudio, permitiendo de esta manera, realizar una aportación científica a los mismos.

A su vez, la utilización de las TICs ha posibilitado la consolidación de redes de investigación que han sido creadas y que mantienen en un elevado nivel de contacto a todas las opciones de comunicación que están disponibles y a la que se accede con relativa facilidad y de manera económica. En este contexto se coincide con Arbelaéz (2014), al señalar que en relación con la gestión de la investigación las TICs propicia el trabajo colaborativo mediante la comunicación sincrónica y asincrónica entre los integrantes de un grupo de investigación, aseguran el almacenamiento en línea de la información, además de posibilitar la comunicación de los resultados de investigación, así como la accesibilidad de estos a la comunidad en general.

Es por todo lo anterior, que el trabajo de los investigadores se beneficia con las herramientas que le ofrecen las TICs, como son el hardware, el software, páginas web, formularios electrónicos y otros, los cuales permiten tener mayor facilidad para el acceso y procesamiento de la información; aspecto esencial y muy útil en las diferentes etapas de la investigación. Siguiendo esta línea Cisneros \& César (2003), alaban las implicaciones particulares que presenta el software para la realización del análisis cualitativo de las investigaciones, por lo que señalan que ello de por sí, constituye una "revolución metodológica". En relación con esto Secrist (2002) indicó que la incorporación de las TICs en las prácticas habituales de investigación constituye un proceso que es considerado como una verdadera "revolución digital". Se comparte con Díaz et al. (2018), al explicar que dentro de las cuantiosas posibilidades que ofrecen las TICs a las investigaciones, está la de propiciar la difusión de los resultados científicos, 
convirtiéndolos en accesibles no solamente a la propia comunidad científica como tal sino de igual manera a la sociedad en general.

En este contexto y en especial en la educación superior, es de gran relevancia la proyección de la metodología de la investigación basada en las TICs. Su integración y aplicación permiten alcanzar con el apoyo de diferentes herramientas didácticas y tecnológicas los objetivos planteados y por ende los resultados esperados. Lo anterior hace se materialicen en una elevada calidad del proceso de enseñanza aprendizaje y en el logro de las competencias a alcanzar por los estudiantes. Atendiendo a lo planteado el objetivo del presente estudio se centra en: Determinar la incidencia de la utilización de las tecnologías de la información y la comunicación en la transformación de la metodología de la investigación científica en el contexto de la educación superior

\section{La metodología de la investigación, su importancia}

La metodología de la investigación, en su definición más general de la literatura de especialidad, es catalogada como el conjunto racional y coherente que posibilita aplicar las técnicas y los procedimientos sistemática y ordenadamente durante la ejecución de una investigación, constituyendo la definitiva la resultante de la forma en que los comprometidos del proyecto investigativo recaben, ordenen y evalúen los datos que obtengan.

Respecto a la metodología como tal, autores como Toala y Mendoza (2019), explican que, como conjunto de procedimientos y técnicas concretas de investigación, cuentan con la posibilidad de que sean aplicadas en cualquier disciplina o área del conocimiento científico.

En relación con ello Trujillo et al. (2004), plantean que la importancia de la metodología de la investigación radica, entre otros aspectos, en que:

- Posibilita la realización de la evaluación reflexiva y crítica de los conceptos teóricos a desarrollar en una investigación.

- Involucra la utilización de tácticas y procedimientos para solucionar problemas con soluciones que se encuentran, aplicando el método científico.

- Permite el desarrollo de la capacidad crítica y el uso del argumento para la toma de decisiones en correspondencia con la continuación de los procesos.

- Constituye una herramienta esencial para desarrollar las actividades académicas y científicas.

- Posibilita el establecimiento de la correcta dirección de una investigación, al asegurar un trabajo eficaz y eficiente para alcanzar los resultados esperados. 
En concordancia con lo anterior, Hernández et al. (2016), destacan que la característica principal y la propia génesis de la universidad moderna, lo constituye la investigación y la formación de una esencia científica en los estudiantes. En esta misma dirección Aguirre y Ruíz (2015), subrayaron que, todo aquello que se conciba para esclarecer y operacionalizar los conceptos y nociones de ciencia, investigación, práctica científica, y otros conceptos referentes, es inseparable a esta tarea.

Se converge con Sánchez (2010), al formular que el desafío está dado, en que el docente que imparte de la asignatura de Metodología de la Investigación Científica debe ser capaz de imaginar y de acudir al acervo de recursos y procedimientos prácticos de su experiencia, de manera que le permita de forma progresiva ir transitando de la enseñanza comunicativa y conceptual a otra más práctica y efectiva del quehacer científico. Apunta que debe ir promoviendo en los estudiantes el aprendizaje científico, lo que propiciará la incorporación de las TICs en la enseñanza y el asesoramiento de procesos investigativos, con una labor tutorial que congregue diferentes saberes: teóricos, prácticos y pedagógicos.

\section{Herramientas TICs utilizadas en el campo de la metodología de la investigación}

Actualmente las TICs brindan escenarios de aprendizaje y socialización nuevos, los cuales se encuentran en una continua transformación en todas sus esferas. El área de la metodología de investigación no escapa a ello, ni tampoco el de las herramientas que cotidianamente propician a los investigadores la realización de su labor.

En relación con lo anterior autores como Colas y Pons (2012), expresan que en análisis realizado a la evolución de las herramientas TICs en relación con la cultura científica, han evidenciado que es indudable que la intervención de las mismas contribuye de manera precipitada al desarrollo y agilidad en la velocidad y cantidad de información que pudiera almacenarse, contribuyendo a la investigación, y para lo cual se toma como referencia al inicio del funcionamiento de herramientas como fueron la internet y aplicaciones como Facebook, twiter, xing, linked-in, Instagram, así como entornos virtuales de enseñanza y aprendizaje que propician acceder a la información y a que se multipliquen las actividades que pueden ser compartidas entre los investigadores.

Por otra parte, Ruiz (2014) expresa que, con el incremento de nuevos espacios y herramientas para la obtención de datos empíricos, al incorporar una cantidad amplia y variada de herramientas, están incluidos tres tecnologías, las cuales se refieren a:

* Las redes de comunicación, que propicia el mantenimiento a distancia del intercambio de información y comunicación, en los que se mezclan imagen y datos. 
* Las bases de datos masivas, que brindan información de manera organizada a la cual puede accederse a través de información de manera selectiva.

* La computación distribuida, esta permite la distribución de los recursos informáticos a través de redes de computadoras.

Respecto a ello autores como Arbeláez (2014), en relación con los dispositivos de almacenamiento virtuales o en la nube, expresa que posibilita al investigador, además de no depender de un espacio físico, contar con información asequible en cualquier lugar donde el mismo se encuentre, sin necesidad de tener su propio computador y al mismo tiempo poder compartir archivos con otros especialistas, mediante herramientas como Google Drive, iCloud y Dropbox entre otros.

\section{Incidencia de las TICs en la transformación de la metodología de la investigación}

En esta dirección uno de los aspectos esenciales en cualquier investigación es el relacionado con el tiempo de realización del propio trabajo investigativo, y en ello, con el apoyo de herramientas TICs se reducen y optimizan los mismos de manera sustancial. Diferentes autores se han referido al tema, tal es el caso de Gross (2016), quien afirma que uno de los factores esenciales del trabajo investigativo lo constituye el hecho de consolidar la forma de conseguir en un tiempo menor los objetivos planteados con la investigación, mediante la creación de una dinámica de trabajo que posibilite planificadamente y con una mayor eficiencia la misma, ello implica que no solo serán importantes las herramientas a utilizar sino además el interaccionar de las personas que desarrollan la investigación.

En este ámbito, autores como Gross (2016), afirman que existen herramientas informáticas que posibilitan el establecimiento entre los investigadores de una significativa agilidad en la ejecución de varias tareas que son esenciales en el ámbito de la investigación y la comunicación, tales como las redes, equipos portátiles, cámaras y videograbadoras, que apoyan en trabajo investigativo.

Es por ello por lo que autores como Pérez (2009), expresan que las TICs constituyen un componente fundamental en la estructuración académico-investigativa virtual, relacionado a la gestión de la información y uso de conocimientos científicos, técnicos y tecnológicos.

En este contexto estudios realizados por diferentes autores, dentro de los que citamos a Pérez y Dressler (2006), sobre la relación entre las tecnologías de la información y Comunicación (TICs) y la gestión del conocimiento, identifican a un conjunto de herramientas y medios que son primordiales en el accionar de los métodos de investigación, tales como:

- intranets, 
- software de simulación y realidad virtual,

- workflow,

- video conferencias,

- datamining 3

- datawarehouse4.

- inteligencia artificial,

- motores de búsqueda,

- mapas de conocimiento.

- páginas amarillas,

- mensajería instantánea y correo electrónico,

- groupware.

Las cuales constituyen herramientas y medios tecnológicos, que cuando se ha evaluado su incidencia dentro del proceso metodológico investigativo, han demostrado que los sistemas de información y comunicación constituyen un eslabón fundamental al integrarse con el conocimiento y su gestión investigativa.

Al respecto, autores como Gamboa y García (2012), destacan que ello hace que sea necesario que se interactúe con diversas aplicaciones que se han desarrollado, en especial desde la implementación de la web 2.0 y la generalización del video digital, lo cual ha creado una nueva cultura investigativa, que ha originado un cambio significativo en las prácticas de investigación.

A ello, otros autores como Berzosa y Arroyo (2016), añaden que también cobra importancia la incorporación de una serie de dispositivos móviles, los que han sido probados en la generación de datos y en el análisis y la representación de ordenadores portátiles, tales como tablets, Smartphone, y teléfonos móviles, que cuentan con diversas aplicaciones como son: CAPI (Computer-Assisted Personal Interviewing), MCAPI (Mobile Computer-Assisted Personal Interviewing), CAI (Computer-Assisted Interviewing, CATI (Computer-Assisted Telephone Interviewing), ACASI (Audio Computer-Assisted Self-Interviewing), T-ACASI (Telephone Audio Computer-Assisted Self-Interviewing), CARI (Computer-Assisted Recorded Interviewing), los cuales se orientan a la recolección de información, esenciales para el análisis de la información cualitativa.

Todo ello ha implicado que en paralelo al desarrollo que alcanzan las tecnologías de la información y la comunicación también se produzca un afianzamiento de los métodos investigativos. Respecto a ello autores como Garzón (2018), afirman que la unión de la tecnología con la investigación y su fortalecimiento en este periodo de desarrollo tecnológico, aparejado a la experiencia adquirida hacen que constituya una parte integral del desarrollo y consolidación de la investigación y del uso de las herramientas informáticas. 
En correspondencia con ello estudios desarrollados respecto al uso de las herramientas TICs en la investigación se han destacado la integración de las diferentes herramientas en línea y fuera de ella, refiriéndose a tres tipos de servicios:

$>$ El apoyo eficiente que brinda para el archivo de datos primarios y la posibilidad del acceso a bases de datos que permite analizar los datos cualitativos.

$>$ La consultoría en cuestiones relacionadas sobre enseñanza e investigación cualitativa.

$>$ Las bases de información, comunicación, publicación, así como, las posibilidades que brinda para la distribución, el manejo y el compartimiento de conocimiento.

Al mismo tiempo, el autor añade que, otro aspecto destacado, relacionado con el uso de las herramientas TICs, está referido a la vinculación a redes que hace que, compartan, coordinen y generen servicios disponibles para construcción de recursos apreciables en el desarrollo de trabajos investigativos ejecutados por investigadores en el mundo, fortaleciendo de esa manera los bancos de datos que existen como soporte para futuras investigaciones a realizarse.

En el mismo orden de ideas Garzón (2018), señala que dentro de las herramientas de mayor relevancia que las TICs han incorporado al servicio de la investigación, se encuentra las bases de datos científicas, que son las que operan información relacionada con artículos científicos, revistas, libros, ponencias y reportes de investigación, tesis y otros trabajos investigativos relevantes, que tienen la posibilidad de ser consultados desde las propias universidades por investigadores y estudiantes que están vinculados a investigaciones que realizan las instituciones académicas.

Es por ello por lo que autores como Escofet et al., (2014), afirman que el conocimiento y utilización de herramientas de las TICs por estudiantes y docentes posibilita la integración y consolidación de la educación en investigación, lo cual según Capanegra et al., (2016), permite que la investigación como tal y la metodología de la investigación en las universidades en conjunto con las empresas se fortalezcan obteniendo resultados más relevantes y de manera más rápida.

En este contexto, diferentes autores han acotado la importancia que revisten las TICs para la optimización de los procesos investigativos. Al respecto Garzón (2018) indica que las TICs constituyen poderosas herramientas que cuentan con la capacidad para optimizar de manera significativa la calidad de la Metodología de la Investigación y específicamente la investigación cualitativa y cuantitativa, impactando de manera positiva en los diferentes criterios de calidad utilizados en la investigación tales como: la credibilidad, transferibilidad, dependencia y confiabilidad. 
Además, el propio autor, expresa que las TICs propician una mejora en los procedimientos que son aplicados en la metodología de la investigación, asegurando la agilidad en el manejo de los datos y ayudando en la calidad científica de los trabajos investigativos que se realizan, lo que se materializa en procesos tales como la recogida mecánica de cuantiosos datos, en la contratación de los resultados con los especialistas o estudiantes que participan en una investigación, en la triangulación ( ya sea de fuentes metodológicas, ) en el muestreo teórico y en las observaciones persistentes o dilatadas en el tiempo.

\section{Herramientas utilizadas en la recogida y análisis posterior de la información}

El tratamiento informático de los datos propicia al investigador crearse de manera rápida y relativamente fácil, de una visión ampliada del objeto de estudio, mayormente dado a la gran cantidad de datos que se pueden ser obtenidos con el apoyo de las nuevas herramientas de recogida de información.

La utilización de técnicas cualitativas o cuantitativas en el momento de obtener la información estará en dependencia del contexto propio de la investigación que se realizará. No obstante, según refieren Campoy y Gómez (2009), el uso de métodos cualitativas, utilizando estas técnicas, han sido objeto de críticas, basadas en una presumible falta de objetividad y validez científica. Ruiz (2011), afirma que actualmente esta situación se ha transformado y fortalecido como procedimiento, dado que intenta conseguir conocimiento científico, dando solución a problemáticas, tales como, el tratamiento de la validez, o el uso de aplicaciones informáticas.

En este contexto, Rodríguez et al. (1999), plantean que la información que brindan las técnicas cualitativas, utilizando estas herramientas, presenta tanta validez como las que aportan las técnicas cuantitativas y que la diferencia se encuentra en el tipo de información que cada una procesa y en que además hay que estar conscientes que no se cuenta con una sola forma de realizar el enfoque de la investigación cualitativa, dado que tenemos distintos enfoques, los que están dados en base a las opciones que se tomen.

Siguiendo esta línea de pensamientos es importante significar que cuando nos referimos a las herramientas de las TICs para la investigación nos estamos refiriendo a aplicaciones informáticas que nos proporcionan la tarea de lograr una reducción de los datos obtenidos en el proceso de investigación. Mientras que en relación con análisis de la información se encuentra disponible un conjunto de software que se utiliza en el análisis de la información cuantitativa, entre los que encontramos el SPSS (Statistical Package for the Social Sciences), Mintab, Stats y Excel. 
En este contexto, Ruiz (2011) describen de manera breve los programas de análisis cualitativo que se emplean con mayor regularidad, entre los que se encuentran:

\section{$\checkmark \underline{\text { Nud.ist }}$}

Constituyen una de las herramientas que más se emplean en la investigación cualitativa. La misma posibilita la agrupación de las categorías de datos y organizarlas conforme a su jerarquía, lo cual permite al investigador evaluar las relaciones existentes entre los diversos conceptos o categorías investigadas.

Al respecto, Gil y Perera (2001) afirman que esta aplicación tolera los procesos de categorización deductiva e inductiva, posibilitando que podamos realizar el diseño de un sistema de categorías desarrollado partiendo de teorías, existentes o establecidas en base al objeto de investigación o también de hipótesis que se han incorporado durante el proceso de recogida de la información.

\section{$\checkmark$ Nud.ist Vivo.}

Esta aplicación presenta un grado superior de avance en relación con la anterior, para la evaluación de datos cualitativos en investigación, y la misma se asocia está a Nud.ist 4.0 o 6.0, constituyendo una de los más utilizadas en el mundo. La misma posibilita realizar la gestión de datos enriquecidos, como textos enriquecidos, empleando negrita, cursivas, colores y otros.

\section{$\checkmark \underline{\text { Atlas.ti }}$}

Esta aplicación informática se utiliza mayormente en la investigación cualitativa o análisis de datos cualitativos, de textos, gráficos, de audios o video. El programa suministra herramientas que posibilitan al usuario localizar el código y tomar notas de los resultados de datos primarios, para medir y analizar su importancia y visualizar las complicadas relaciones entre los mismos. De igual manera, esta aplicación posibilita realizar la gestión y el análisis de elevados volúmenes de documentos y realiza un seguimiento de todas las anotaciones y códigos en todas las esferas que requieran un estudio riguroso.

\section{$\checkmark$ Aquad}

La característica fundamental de esta aplicación es la facilidad que tiene para el investigador de extraer conclusiones cuando se relacionan las categorías entre ellas, evaluando la aparición de determinadas configuraciones típicas y reiterativas en la representación de datos. 


\section{$\checkmark$ Ethnograph}

Esta aplicación está diseñada para realizar análisis de datos recogidos durante la ejecución de las investigaciones cualitativas, de forma sencilla y eficiente. La misma posibilita la importación de archivos planos o escritos en cualquier procesador de textos, de manera directa al programa. La aplicación propicia la tarea del investigador cuando pretendemos encontrar y resaltar fragmentos de interés del conjunto de datos, marcarlos con códigos y efectuar análisis que son disponibles a ser recuperados para incluirlos en análisis posteriores.

\section{$\checkmark$ LimeSurvey.}

Es un software libre, que permite la elaboración de cualquier encuesta posible, con varias funcionalidades y características para la obtención de resultados óptimos. Con el mismo se posibilita que los resultados sean anónimos, separando los datos de los participantes de los datos que suministran, inclusive en encuestas que sean controladas

Actualmente, diferentes autores expertos, entre los que citamos a Ruiz (2011) la consideran como la herramienta más utilizada online, entre aquellas herramientas libres y gratuitas existentes.

La herramienta permite la realización de preguntas opcionales, obligatorias, con posibilidades de una o varias respuestas y preguntas condicionadas, lo que origina que la misma se presente muy poderosa para la realización de las investigaciones. Por otra parte, son de mucha utilidad los informes que con ella se generan, dado que posibilita la exportación de datos (hoja de cálculo, hoja de texto, SPSS y otros), y permite la generación de gráficas filtradas de acuerdo con las necesidades que requiera.

\section{Situaciones adversas que pueden generar el uso de las TICs como herramienta de} la metodología de investigación

La utilización de las TICs como herramientas en la Metodología de la Investigación incorporan de igual manera aspectos negativos, tales como el de priorizar el aspecto tecnológico de una investigación sobre el aspecto metodológico, lo cual debe ser conocido y sopesado por el investigador, quien deberá de manera permanente solventar las posibles situaciones que puedan presentarse al respecto.

Referente a este tema, Arbeláez (2014) expone que existe el riesgo por parte del investigador de confundir el medio que facilita la investigación con el objetivo de la misma, o sea, pensar que las herramientas que proveen las TICs al investigador son un fin en sí misma y no un medio para dar solución a situaciones inherentes al propio proceso de investigación, sin tomar en consideración las posibilidades y riesgos en que 
puede incurrir el mismo, cuando enfoca su atención en el uso de las herramientas tecnológicas por encima del análisis metodológico de la investigación.

Destaca el propio autor que las diferentes ventajas que incorpora las TICs a la Metodología de la Investigación, expresadas con anterioridad, crea unas expectativas elevadas en el investigador, relacionadas especialmente con la calidad de los resultados que obtendrá como producto final. No obstante, las mismas pueden desvanecerse, si el mismo no tiene claridad respecto a sus potencialidades y limitaciones.

En este sentido Flick (2007), señaló que las repercusiones de la utilización de las Tecnologías de la Información y Comunicación en la investigación, deben reflexionarse por los propios investigadores, mediante preguntas en relación con el tipo de datos que requiere el programa, sobre las actividades específicas que pueden realizarse con las herramientas utilizadas, sobre el proceso propiamente relativo a los aspectos de la recogida o análisis de la información que provee el programa, sobre las técnicas, o sea, lo que se requiere en hardware, sobre los costos y tiempos de procesamiento así como, sobre las competencias profesionales que se requieren del investigador, o sea, sus destrezas, conocimientos técnicos sobre el tema de investigación y otros.

Estudios realizados por Cisneros y César (2003), sobre análisis cualitativos de datos asistidos por computadoras, evidenciaron que su aplicación no había sido realizada con una reflexión crítica sobre la generación del dato. Ello contrapone lo expresado por Coffey \& Atkinson (1996) al explicar que no existe ningún programa de computadora que realice automáticamente el análisis de datos. Ellos son dependientes de lo que los investigadores definan como las temáticas analíticas a ser exploradas, las ideas que son fundamentales y los modos de representación que son más apropiados.

\section{Metodología}

En el estudio se utilizó una metodología descriptiva, basada en métodos teóricos dentro de estos el histórico-lógico, analítico -sintético, inductivo-deductivo y la revisión documental, lo cual permitió realizar la revisión de la literatura precedente y establecer los argumentos esenciales relacionados con el tema. Se establecieron criterios referentes para la búsqueda dentro de estos: Las herramientas TICs utilizadas en el campo de la metodología de la investigación, la incidencia de las TICs en la transformación de la metodología de la investigación, las herramientas utilizadas en la recogida y análisis posterior de la información, así como se esbozaron las situaciones adversas que pueden generar el uso de las TICs como herramienta de la Metodología de Investigación, lo cual propició la calidad del trabajo realizado.

\section{Resultados y Discusión}


El análisis de los referentes teóricos consultados nos permite establecer los estudios realizados por diversos autores, tales como Sánchez (2010), plantean que el desafío de la investigación en la universidad, está dado, en que el docente que imparte la asignatura de metodología de la investigación científica, debe ser capaz de imaginar y de acudir al acervo de recursos y procedimientos prácticos de su experiencia, de manera que le permita de forma progresiva ir transitando de la enseñanza comunicativa y conceptual a otra más práctica y efectiva del quehacer científico, promoviendo en los estudiantes el aprendizaje científico, lo que propiciará la incorporación de las TICs en la enseñanza y el asesoramiento de procesos investigativos, con una labor tutorial que congregue diferentes saberes: teóricos prácticos y pedagógicos.

En correspondencia con lo señalado se concuerda con Gross (2016), al afirman que uno de los factores esenciales del trabajo investigativo en el contexto de la Educación Superior con el apoyo de las herramientas TICs, lo constituye el hecho de consolidar la forma de conseguir en un tiempo menor los objetivos planteados con la investigación, mediante la creación de una dinámica de trabajo que posibilite planificadamente y con una mayor eficiencia la misma, ello implica que no solo serán importantes las herramientas a utilizar sino además el interaccionar de las personas que desarrollan la investigación.

Esto hace sustentar la importancia y utilidad de las TICs en las investigaciones destacando que estas constituyen poderosas herramientas que permiten optimizar y transformar de manera significativa la calidad de la Metodología de la Investigación y específicamente la investigación cualitativa y cuantitativa desarrolladas en el contexto de la educación Superior. Esto ha provocado se logre impactar de forma positiva en los diferentes criterios de calidad utilizados en la investigación tales como: la credibilidad, transferibilidad, dependencia y confiabilidad (Garzón, 2018).

Por otra parte resulta fundamental destacar se ha evidenciado en la revisión realizada que contrariamente al criterio muchas veces generalizado sobre la información que brindan las técnicas cualitativas, utilizando las herramientas TICs, sobre su validez y fiabilidad se demuestra que son tan validas como los resultados que se aportan derivados de las técnicas cuantitativas; concordándose con Rodríguez et al. (1999), al señalar que la diferencia se encuentra en el tipo de información que cada una procesa y en que además hay que estar conscientes que no se cuenta con una sola forma de direccionar la investigación dado que tenemos distintos enfoques, los que cuales quedan determinados basado en las opciones que se tomen.

De igual manera cabe desatacar que la utilización de las TICs como herramientas a utilizar en el contexto de la metodología de la investigación pueden estar sujetas a caer en aspectos negativos, tales como el de priorizar el aspecto tecnológico de una investigación sobre el aspecto metodológico. Al respecto se coincide con Arbeláez 
(2014), al enfatizar en la idea de que existe el riesgo por parte del investigador de confundir que las herramientas que proveen las TICs son un fin en sí misma y no un medio para dar solución a situaciones inherentes al propio proceso de investigación, originándose ello, cuando enfoca su atención en el uso de las herramientas tecnológicas por encima del análisis metodológico de la investigación; es por ello tan importante su control y seguimiento.

Derivado de todo lo analizado se asume por parte de los autores de la presente investigación cuatro resultados esenciales a destacar:

- Se evidencia que las TICs constituyen herramientas capaces de optimizar significativamente la calidad de la Metodología de la Investigación en el contexto de la Educación Superior, su empleo permite conseguir en menor tiempo y con la calidad requerida los objetivos planteados, creando espacios para el intercambio y la generación de soluciones.

- Se demuestra que el desafío de la investigación en la universidad radica en el rol del docente que imparte la asignatura de Metodología de la Investigación, quien deberá tener las competencias que le permitan realizar una labor tutorial que congregue los saberes: teóricos, prácticos y pedagógicos y la interacción entre los investigadores.

- Se sustenta que la información que brindan las técnicas cualitativas, utilizando las herramientas TICs, presentan tanta validez como las que aportan las técnicas cuantitativas, garantizando su calidad en cuanto a criterios tales como: credibilidad, transferibilidad, dependencia y confiabilidad.

- Es necesario enfatizar en la premisa de que el empleo indiscriminado de las TICs trae consigo ciertos riesgos, resultado fundamental su control y seguimiento para evitar que el investigador enfoque su atención en el uso de las herramientas tecnológicas por encima del análisis metodológico de la investigación.

\section{Conclusiones}

- La Revisión Sistemática de la Literatura, (RSL), determinó el alto grado de incidencia que tiene la utilización de las Tecnologías de la Información y Comunicación (TICs) como herramienta fundamental a utilizar en el contexto de la Metodología de la Investigación, con énfasis en la Educación Superior. Su empleo constituye una revolución digital y metodológica de las investigaciones, materializándose en elevada calidad del proceso de enseñanza aprendizaje y en las competencias logradas por los estudiantes, lo cual permite consolidar la 
forma de conseguir en un tiempo menor y con la calidad requerida los objetivos planteados en la investigación y la creación de una dinámica de trabajo que posibilita su desarrollo de forma planificada y con una mayor eficiencia en cuanto a la interacción de los investigadores y al cumplimiento de los criterios de calidad.

- De igual manera, quedaron comprobados los riesgos que trae la utilización indiscriminada de las TICs en el contexto de la Metodología de la Investigación; precisándose que esto puede darse si los investigadores hacen prevalecer en el proceso investigativo el uso de las herramientas tecnológicas sobre el análisis metodológico derivado de la propia la investigación, lo cual requiere de su control permanente.

\section{Referencias Bibliográficas}

Aguirre, G. \& Ruíz, R. (2015). TICs en el apoyo a la enseñanza de la investigación y las asesorías de tesis. En: Ventana Informática No. 32 (ene-jun). Manizales (Colombia): Facultad de Ciencias e Ingeniería, Universidad de Manizales. p. 1127.

ISSN:

0123-

9678.https://revistasum.umanizales.edu.co/ojs/index.php/ventanainformatica/arti cle/view/1094/1198.

Arbeláez, M. (2014). Las tecnologías de la información y la comunicación (TIC) un instrumento para la investigación. Investigaciones Andina, vol.16 no.29 Pereira Julio 2014. Print versión ISSN 0124-8146. http://www.scielo.org.co/scielo.php?script=sci_arttext\&pid=S012481462014000200001

Berzosa, I. \& Arroyo, M. (2016). Docentes y TIC: un encuentro necesario. Contextos educativos (19), $147 \quad-\quad 159 . \quad$ doi:10.18172/con.2767. https://redib.org/Record/oai_articulo1608850-docentes-y-tic-un-encuentronecesario

Campoy, T. \& Gomes, E. (2009). Técnicas e instrumentos cualitativos de recogida de datos. En Pantoja, A. (Coord.). Manual básico para la realización de tesinas, tesis y trabajos de investigación (pp.275-302). Madrid: EOS Universitaria. https://revista.uisrael.edu.ec/index.php/rcui/article/view/400/218

Capanegra, H. A., Cabrera, G., Aguilar, M. L., \& Jorda, M. S. (2016). El empleo de las tecnologías de información y comunicaciones (TICs) en el ámbito universitario. Documentos y Aportes en Administración Pública y Gestión Estatal, 16(26), 159-190. Obtenido de http://www.redalyc.org/articulo.oa?id=337546668006. 
Cisneros, P. \& César, A. (2003). "Análisis cualitativo asistido por computadora. Sociologías, vol. 5, núm. 9, enero-junio, 2003, pp. 286-313 Universidad Federal do Rio Grande do Sul Porto Alegre, Brasil. https://www.redalyc.org/pdf/868/86819565014.pdf.

Coffey, A. \& Atkinson, P. (1996). Making sense of qualitative data. Complementary Research Strategies Thousand oaks, CA: sage. ISBN: 9780803970533. https://us.sagepub.com/en-us/nam/making-sense-of-qualitative-data/book5617.

Colas, P. \& Pons, J. (2012). Aplicaciones de las Tecnologías de la Información y la Comunicación en la Investigación Cualitativa. Revista Española de Pedagogía, $\begin{array}{llll}\text { Año LXX, } & \text { Nro. } & \text { 77-92. }\end{array}$ https://www.academia.edu/4399018/Aplicaciones_de_las_tecnologias_de_la_inf ormacion_y_la_comunicacion_en_la_investigacion_cualitativa

Díaz, E., Díaz, J., Gorgoso, A., Sánchez, Y., Riverón, G. \& Santiesteban, D. (2018). Presencia de las TIC en las investigaciones sociales. RITI Jornal, Vol. 6, 11 (enero-junio 2018). ISSN: 2387-0893.file:///C:/Users/USER/Downloads/DialnetPresenciaDeLasTICEnLasInvestigacionesSociales-7107374.pdf

Escofet, A., López, M. \& Álvarez, G. (2014). Una mirada crítica sobre los nativos digitales: análisis de los usos formales de TIC entre estudiantes universitarios. Revista Q, 9(17), $1 \quad$ - $18 . \quad$ Obtenido de: https://www.researchgate.net/publication/286454234_Una_mirada_critica_sobre _los_nativos_digitales_Analisis_de_los_usos_formales_de_TIC_entre_estudiant es_universitarios_A_Critical_View_of_Digital_Natives_Analysis_of_formal_st udent_uses_of_ICT

Flick, U. (2007). Introducción a la investigación cualitativa. 2da Edición. Ediciones Morata, S. L (2007). ISBN: 978-84-7112-480-7. http://investigacionsocial.sociales.uba.ar/wpcontent/uploads/sites/103/2013/03/INVESTIGACIONCUALITATIVAFLICK.p df

Gamboa, M. \& García, Y. (2012). Aprender haciendo en Investigación como estrategia de aprendizaje. Revista de investigaciones UNAD (2), 77 - 93. http://search.ebscohost.com/login.aspx?direct=true \&db=fap\&AN=94059360\&la ng=es\&sit e=ehost-live.https://hemeroteca.unad.edu.co/index.php/revista-deinvestigaciones-unad/article/view/789/0. 
Garzón, L. (2018). Uso y apropiación de herramientas TIC de estudiantes y docentes investigadores en universidades en Medellín. Revista Psico espacios, Vol. 12, N. 20, pp.49-73. Disponible en https://doi.org/10.25057/issn.2145-2776 file:///C:/Users/User/Downloads/Dialnet-

UsoYApropiacionDeHerramientasTICDeEstudiantesYDoce-6573536.pdf.

Gil, J. \& Perera, V. (2001). Análisis informatizado de datos cualitativos. introducción al uso del programa NUD.IST-5. Sevilla: Editorial Kronos. ISBN: 84-86273-28-5. https://dialnet.unirioja.es/servlet/libro?codigo=175410.

Gross, B. (2016). Retos y tendencias sobre el futuro de la investigación acerca del aprendizaje con tecnologías digitales. Revista de Educación a Distancia RED (50), 2 - 2 13. Doi: http://dx.doi.org/10.6018/red/50/10. https://www.um.es/ead/red/32/gros.pdf

Hernández, R., Fernández, C. \& Baptista, M. (2016). Metodología de la investigación. 6ta edición. Editora McGraw-Hill / Interamericana Editores, S.A. DE C.V. ISBN: $\quad$ 978-1-4562-2396-0. http://observatorio.epacartagena.gov.co/wpcontent/uploads/2017/08/metodologia-de-la-investigacion-sextaedicion.compressed.pdf

Pérez, D. \& Dressler, M. (2006). Tecnologías de la información para la gestión del. Intangible Capital, N. ${ }^{\circ} 15$ - Vol. 3- pp. 31-59, Ene-Mar de 2007 - ISSN: 16979818 (Cod:0075) Obtenido de: https://biblioteca.udgvirtual.udg.mx/jspui/bitstream/123456789/906/3/Tecnolog \%c3\%adas_informaci\%c3\%b3n_gesti\%c3\%b3n.pdf.

Pérez, M. (2009). La comunicación y la interacción en contextos virtuales de aprendizaje. Revista apertura. UDGVIRTUAL. e- ISSN 2007-1094. Universidad de Guadalajara. https://www.udgvirtual.udg.mx/apertura/index.php/apertura/article/view/15/18.

Ruiz, J. (2011). Herramientas para la investigación en tecnologías de la información y la comunicación. casos de estudio. Profesorado, Revista de currículo y formación de profesorado. VOL. 15, N. ${ }^{\circ} 1$ (2011). ISSN 1138-414X (edición papel). ISSN 1989-639X (edición electrónica). http://www.ugr.es/ recfpro/rev151ART9.pdf.

Ruiz, C. (2014). Evaluación de una experiencia de tutoría virtual de tesis de grado en el contexto de un programa de doctorado en educación. Paradigma, 35(1), 129-148. Obtenido de http://ve.scielo.org/pdf/pdg/v35n1/art06.pdf. 
Rodríguez, G., Gil, G., García, J. \& García, E. (1999). "La entrevista” en Metodología de la investigación educativa. Ediciones Málaga, Aljibe, pp. 167-184. https://iessb.files.wordpress.com/2015/03/rodrguez-y-otros-1999-laentrevista.pdf

Sánchez, R. (2010). Enseñar a investigar: Una didáctica nueva de la investigación en ciencias sociales y humanas. 3 ed. México (México): Plaza y Valdés. Colección. Educación superior contemporánea. 190 p. ISBN: 978-607-402-236-0. https://www.fceia.unr.edu.ar/geii/maestria/2014/DraSanjurjo/8mas/Ricardo\%20 Sanchez\%20Puentes,Ense\%C3\%B1ar\%20a\%20investigar.pdf.

Secrist, C. (2002), "Combining Digital Video Technology and Narrative Methods for Understanding Infant Development", an Forum Qualitative Social Research, vol. 3, mum. 2. https://www.proquest.com/docview/867759785

Toala, G. \& Mendoza, A. (2019). Importancia de la enseñanza de la metodología de la investigación científica en las ciencias administrativas. Dom. Cien., ISSN: $2477-$ 8818 Vol. 5, núm. 2, abril 2019, pp. 56-70. file:///C:/Users/USER/Downloads/DialnetImportanciaDeLaEnsenanzaDeLaMetodologiaDeLaInvesti-6989278\%20(2).pdf.

Trujillo, G., Zambrano, D. \& Vargas, A. (2004). Importancia de una metodología de investigación y su relación con el diseño y elaboración de un proyecto investigativo de carácter http://catarina.udlap.mx/u_dl_a/tales/documentos/lhr/castaneda_c_ga/capitulo3.p df

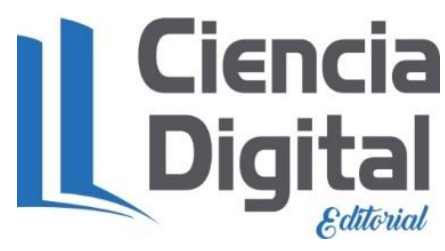


El artículo que se publica es de exclusiva responsabilidad de los autores y no necesariamente reflejan el pensamiento de la Revista Alfa Publicaciones.

\section{Ciencia}

El artículo queda en propiedad de la revista y, por tanto, su publicación parcial y/o total en otro medio tiene que ser autorizado por el director de la Revista Alfa Publicaciones.
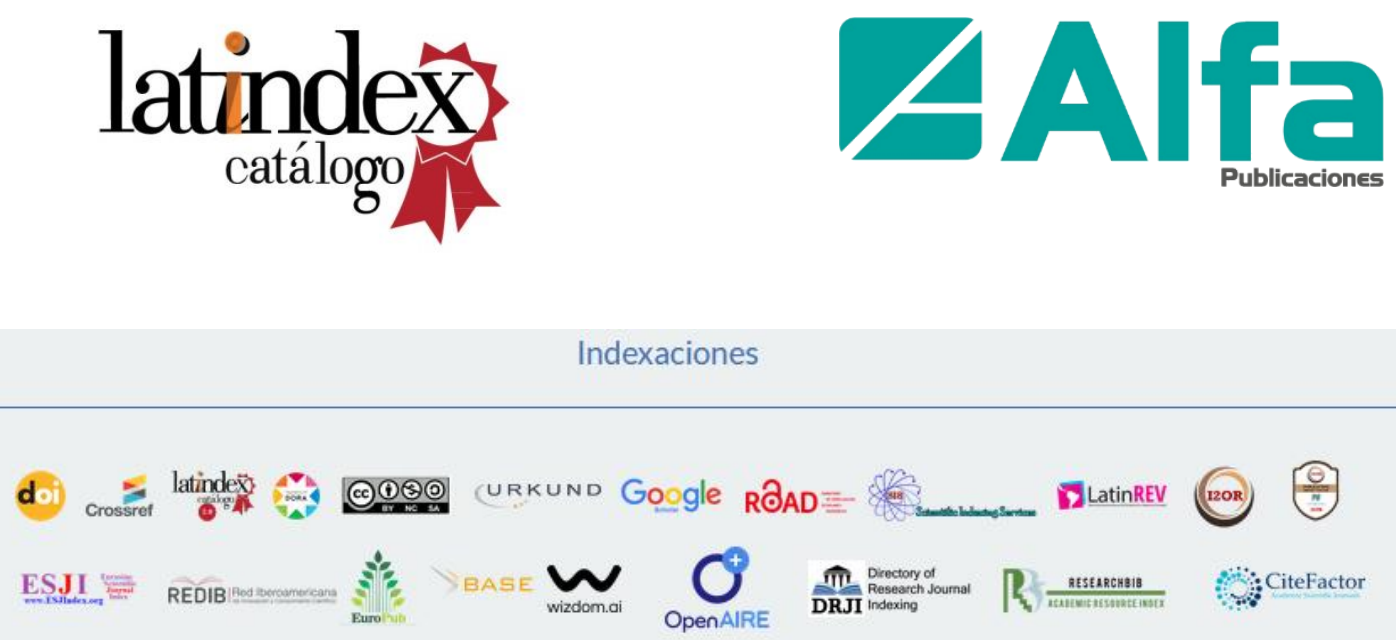